\title{
Value of Nitrosourea-based Chemotherapy in Recurrent High Grade Glioma, NEMROCK Experience
}

\author{
Hisham Atef, Amr Sakr, Ashraf Mounir and Ayah Hussein \\ Kasr Al-Aini Center of Clinical Oncology and Nuclear Medicine (NEMROCK), Faculty of Medicine, \\ Cairo University
}

Background: The prognosis of high-grade gliomas is generally poor, There is controversy about the potential benefits of treatment when the tumor progress. The main idea of this study is the assessment of the value of nitrosourea-based chemotherapy in recurrent high grade glioma. Overall survival was the primary endpoint. Patients and Methods: between the period of January 2007 till December 2011, 112 cases with progressive high grade glioma were recruited from Kasr-Al Ainy Center of Clinical Oncology and Nuclear Medicine (NEMROCK), Faculty of Medicine, Cairo University.

Results: out of 112 cases, $59(52.6 \%)$ were for patients received Post progression nitrosourea- based chemotherapy, while $53(47.4 \%)$ were for patients received best supportive care only. Three patients had partial response (5\%), 16 patients had stable disease (27\%). Median Progression free survival of patients receiving nitrosourea- based chemotherapy was 3 months, with 6-month PFS of 22\%. Median overall survival was 7 months in chemotherapy group and 4 months in best supportive care group with a statistical significance $(P$ Value $<0.001)$.

Conclusion: The study showed a clear difference in overall survival in patients receiving nitrosourea-based chemotherapy from those receiving best supportive care.

Key words: high grade glioma, anaplastic astrocytoma, glioblastoma multiform, nitrosourea

Corresponding Author: Amr Sakr

E-mail: amrsakr@yahoo.com

\section{INTRODUCTION}

High grade glioma still has poor prognosis. The standard treatment consists of maximal surgical resection followed by radiotherapy concomitant with temozolomide followed by 6 months temozolomide. The maximum median survival of glioblastoma multiform (GBM) is 14.6 months $^{1}$ and 21.4 months for anaplastic astrocytoma (AA) $)^{2}$.

With standard treatment, nearly all malignant gliomas will recur; usually within one to two years following diagnosis. There is controversy regarding the potential benefits of treatment when the tumor progresses if treatment is considered, treatment of a high-grade glioma that has progressed does not always improve survival compared to supportive care alone ${ }^{3,4}$. With a median survival of about 6.7 months by combination chemotherapy (Procarbazine, Lomustine, and Vincristine) and only 7.2 months with Temozolomide ${ }^{5}$. Bevacizumab alone or in combination with irinotecan is a very active choice, median overall survival were 9.2 months and 8.7months, respectively. However; it is associated with potentially serious side effects like hypertension, thromboembolism and it is an expensive drug $^{6}$.
In our institution, Lomustine plus Vincristine is the second line palliative treatment. This combination of nitrosourea based chemotherapy has several advantages mainly it is relatively cheap, available, effective with minimal toxicity.

The main objective of this study is to assess the value of nitrosourea-based chemotherapy in the treatment of recurrent high grade glioma. Retrospective analysis of the cases presented to Kasr Alaini Center of Clinical Oncology and Radiation Therapy (NEMROCK) was done. The primary end point is overall survival. Secondary end points include response rate, progression free survival and 6-month progression free survival.

\section{PATIENTS AND METHODS}

The current study is a retrospective one carried out at Kasr-Al Ainy Center of Oncology and Nuclear Medicine (NEMROCK), Faculty of Medicine, Cairo University between January 2007 and December 2011. Inclusive one hundred and twelve medical records with progressive high grade glioma were recruited to be analyzed. 


\section{Patient Eligibility}

To be considered eligible for this study, patient's age at the time of primary diagnosis ranged between 18 years and 65years, histopathological diagnosis of high grade glioma (anaplastic astrocytoma or glioblastoma multiform), previous irradiation to primary brain tumor with or without concomitant Temozolomide, and radiological evidence of disease progression confirmed by magnetic resonance imaging (MRI). Patients who did not receive radiotherapy as a primary treatment after diagnosis were excluded.

Patient's records were reviewed for: age, performance status using Karnofsky Performance status (KPS) score (good performance $>70$ or bad performance $\leq 70$ ), Extent of surgery (either stereotactic biopsy, partial or total excision), Time from first diagnosis to progression, Histological subtype was retrieved from the pathological reports of either excisional or stereotactic biopsies.

\section{Treatment modalities:}

The primary treatment was surgery (stereotactic biopsy or partial excision) followed by external irradiation $\{60$ Gray in 30 fractions over 6 weeks $\}$ with or without concomitant Temozolomide. Disease progression was diagnosed radiologically by contrastenhanced MRI. After progression, a group of patients received chemotherapy and another group received best supportive care alone.

\section{Chemotherapy given:}

Lomustin $110 \mathrm{mg} / \mathrm{m}^{2}$ orally D1 and Vincristin $1.4 \mathrm{mg} / \mathrm{m}^{2}$ (Maximum 2mg) intravenous D1\&D21, cycle repeated every 6 weeks.

\section{Response assessment:}

Baseline contrast-enhanced MRI examination was performed before administration of chemotherapy. Subsequent evaluations were carried out every two to three cycles during chemotherapy. Response was scored as a complete response (CR), partial response $(\mathrm{PR})$, stable disease (SD), or progressive disease (PD). Response assessment was based on Macdonald criteria with the recent RANO (Response Assessment in Neuro-Oncology) modification ${ }^{7}$. Objective response was defined as decreased or no enhancement on the post-contrast T1 images and stability or improvement on fluid-attenuated inversion recovery $\mathrm{T} 2$ images. Only contrast-enhancing lesions were measured. Non-contrast-enhancing lesions were considered nontarget lesions in tumor assessment. Progression was determined by contrast-enhancing and non-contrastenhancing lesions. Any new area of non-enhancing $\mathrm{T} 2$ or fluid-attenuated inversion-recovery signal consistent with tumor was considered progressive disease. In the absence of radiographic documentation, clinical progression was used to determine progression.

Duration of response was measured from the date when the criteria were first met for response until the first date of progressive disease or death from any cause, whichever occurred first.

To address the issue of pseudoprogression, which is prevalent within the first 12 weeks of completion of radiotherapy, progression can only be determined if the majority of the new enhancement is outside of the radiation field (for example, beyond the high-dose region or $80 \%$ isodose line) or if there is pathologic confirmation of progressive disease.

\section{Statistical analysis:}

Progression Free Survival period was calculated as the interval from the date of documented proved progression radiologically until second progression either radiologically or clinically if scan cannot be performed or death or last follow-up. Overall Survival period was measured as the interval between the date of documented proved progression radiologically until death or date of the last follow-up.

Data were analyzed using SPSS win statistical package version 17 (SPSS Inc., Chicago, IL). Numerical data were expressed as mean and standard deviation or median and range as appropriate. Qualitative data were expressed as frequency and percentage. Chi-square test (Fisher's exact test) was used to examine the relation between qualitative variables. Survival analysis was done using KaplanMeier method and comparison between two survival curves is done using log-rank test. A $P$-value $\leq 0.05$ was considered significant.

\section{RESULTS}

From January 2007 till December 2011 inclusive medical records of 112 cases with progressive high grade glioma were reviewed. 59 patients received nitrosourea based chemotherapy and 53 patients received best supportive care (BSC). All patients in our study had either stereotactic biopsy or partial excision $\{R(2)$ surgery $\}$ as a diagnostic or primary treatment intervention, followed by external beam radiotherapy 60 Gray over 30 fractions over six weeks. Fourteen patients with glioblastoma multiform received Temozolomide concomitant with radiotherapy and as adjuvant treatment for 6 months. Patients' characteristics are listed in Table (1). For the 59 patients who received nitrosourea 
based chemotherapy, the median number of cycle received were two ranging from one cycle up to eight cycles. Thirteen patients received only one cycle, twenty three patients received two cycles and also twenty three patients received more than two cycles.

Response evaluation was performed on 50 patients (50 patients out of 59 patients $84.7 \%$ ). There was no CR, three patients had PR (5\%), 16 patients had SD (27\%), while 31 patients had PD (52.5\%). The three patients with PR were of anaplastic astrocytoma group. Duration of response ranged from 4 to 9 months.

For nitrosourea based chemotherapy group, median progression free survival was 3 months with 6-month PFS of $22 \%$.

For all patients with high grade glioma, Nitrosoureabased Chemotherapy had a median overall survival of 7 months (95\% 5.89 to 8.01 ) versus 4 months overall survival ( $95 \% 3.37$ to 4.62$)$ for patients who received best supportive care (BSC) with statistical significance $(P$ Value $<0.001)$ as shown in Figure (1). For AA patients who received chemotherapy had a median overall survival of 8 months $(95 \% 6.72$ to 9.27), while patients receiving $\mathrm{BSC}$ had 6 months median overall survival ( $95 \% 4.76$ to 7.23$)$, as shown in Figure (2), with statistical significance $(P$ value $<$ $0.001)$. GBM patients who received chemotherapy had median overall survival 6 months (95\% 4.79 to 7.21), while GBM patients receiving BSC had 3 months median overall survival (95\% 2.3 to 3.6 ), as shown in Figure (3), with statistical significance $(P$ value $<0.001)$

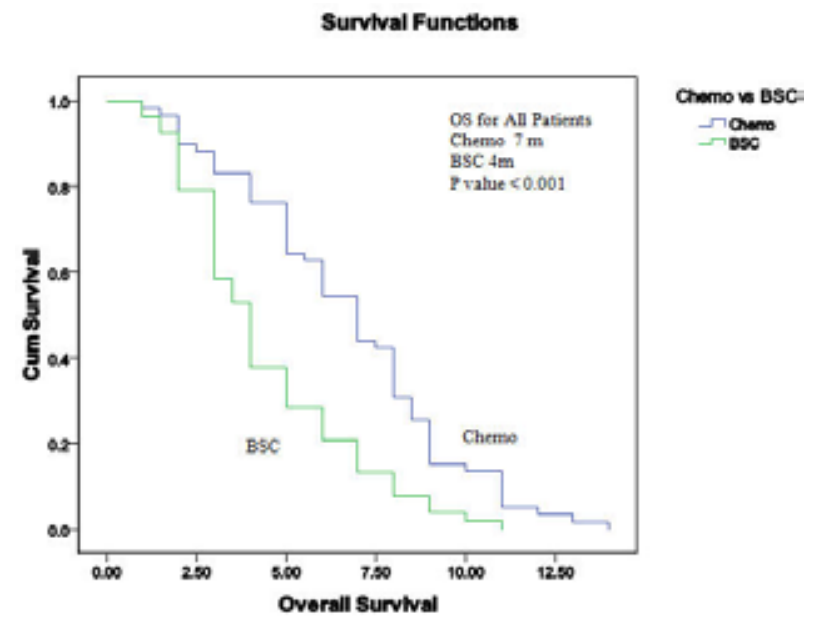

Figure 1: Kaplan-Meier curve of overall Survival for all patients (chemotherapy versus BSC).

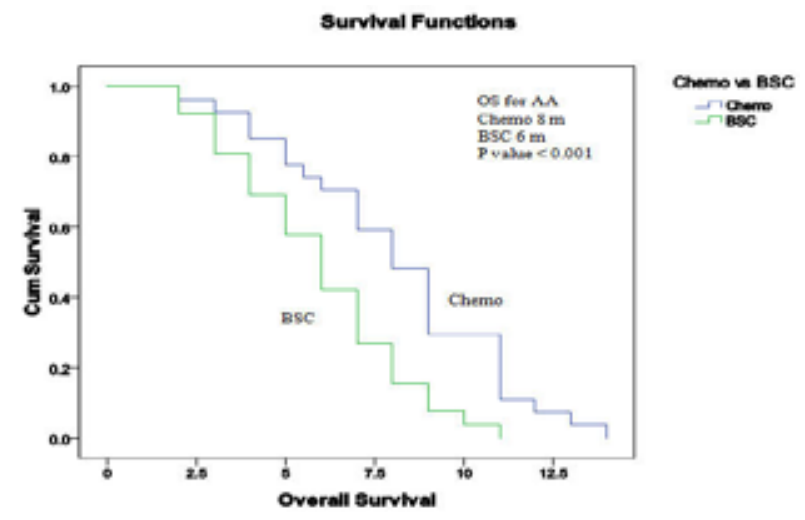

Figure 2: Kaplan-Meier curve of overall Survival for Anaplastic Astrocytoma patients (chemotherapy versus BSC).

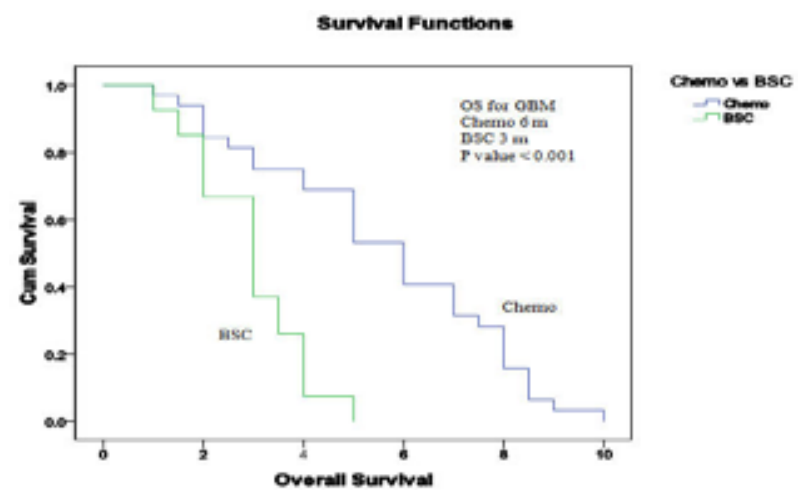

Figure 3: Kaplan-Meier curve of overall Survival for Glioblastoma multiform patients (chemotherapy versus BSC).

Table 1: Descriptive analysis of patients characteristics at first presentation $(N=112)$.

\begin{tabular}{|c|c|c|}
\hline Characteristic & $\begin{array}{c}\text { Nitrosourea } \\
\text { based } \\
\text { chemotherapy } \\
59 \text { patients }\end{array}$ & $\begin{array}{c}\text { BSC } \\
53 \text { patients }\end{array}$ \\
\hline \multicolumn{3}{|l|}{ Age, years } \\
\hline Median & 54 & 56 \\
\hline Range & $22-70$ & $26-73$ \\
\hline \multicolumn{3}{|l|}{ Gender } \\
\hline Male & $40(67.7 \%)$ & $38(71.6 \%)$ \\
\hline Female & $19(32.2 \%)$ & $15(28.3 \%)$ \\
\hline \multicolumn{3}{|l|}{ Histology } \\
\hline $\mathrm{AA}$ & $27(45.7 \%)$ & $26(49.1 \%)$ \\
\hline GBM & $32(54.2 \%)$ & $27(50.9 \%)$ \\
\hline \multicolumn{3}{|l|}{ KPS } \\
\hline High, 90 or 100 & $22(37.2 \%)$ & $17(32.1 \%)$ \\
\hline Low, 70 or 80 & $37(62.7 \%)$ & $36(67.9 \%)$ \\
\hline \multicolumn{3}{|c|}{ Type of Surgery at diagnosis } \\
\hline Biopsy & $21(35.5 \%)$ & $18(33.9 \%)$ \\
\hline Partial excision & $38(64.4 \%)$ & $35(66.1 \%)$ \\
\hline \multicolumn{3}{|c|}{$\begin{array}{l}\text { Time from first diagnosis to } \\
\text { progression, months }\end{array}$} \\
\hline Median & 9 & 10 \\
\hline Range & $3-25$ & $2-26$ \\
\hline Previous Temozolomide & $10(16.9 \%)$ & $4(7.5 \%)$ \\
\hline
\end{tabular}




\section{DISCUSSION}

Management of recurrent high grade glioma is a very hard task. We have limited options, started from palliative surgery to re-irradiation to chemotherapy and end with best supportive care. Surgical resection is the best option especially for those with a mass effect; it provides rapid relief of symptoms. Patients in whom the volume of residual disease was smaller than $10 \mathrm{~cm}^{3}$ had an improved 1-year survival rate of $37 \%$ compared with $18 \%$ for patients with a volume larger than $15 \mathrm{~cm}^{3}$; however it is only applied for patients with good performance status and peripheral lesions ${ }^{8,9}$. Single-fraction Stereotactic radiosurgery is a very attractive option for small lesion with a median survival of about 10 months. However it is only applied for small percentage of patients with small lesions, and taking the results of the Radiation Therapy Oncology Group (RTOG) 93-05 trial, which suggest that the addition of SRS to newly diagnosed GBM does not improve survival, as it indicate the limited benefit of SRS in infiltrating glioma ${ }^{10,11}$. Also, the same principle to brachytherapy which requires expert team. Accordingly, most of patients required palliative chemotherapy.

The choice of chemotherapy is a very important issue; first, we still have no established chemotherapy regimen available as standard, we have a wide range of active drugs starting from bevacizumab-based chemotherapy, temozolomide, nitrosourea-based chemotherapy and platinum based regimens. Here, in this setting the aim is palliative. So we have to consider many factors like cost of the drug, drug efficacy, performance status of patients and toxicity of the drug. Bevacizumab alone or in combination with irinotecan is an active regimen, median overall survival times were 9.2 months and 8.7 months, respectively. However; it is associated with potentially serious side effects like hypertension, thromboembolism and it is an expensive $\operatorname{drug}^{6}$.

Temozolomide (TMZ) is a good choice, it is well tolerated, has good oral bioavailability, and is convenient to administer as an outpatient regimen however, it is relatively expensive drug, and usually it is used as primary treatment in combination with radiotherapy ${ }^{12}$. Rechallenge with $\mathrm{TMZ}$ at progression is a matter of debate, date are conflicting ${ }^{13,14,15}$. Rechallenge with continuous dose-intense TMZ $50 \mathrm{mg} / \mathrm{m}^{2} / \mathrm{d}$ for GBM were patients divided into three groups according to the timing of progression during adjuvant therapy \{early (GBM patients failing during the first 3-6 months of adjuvant therapy) [B1], extended (GBM patients failing after more than 6 months of therapy) [B2], and rechallenge (GBM patients who recurred after stopping treatment) $[\mathrm{B} 3]\}$. Median PFS were 3.6, 1.8, and 3.7 months, respectively ${ }^{14}$.
Nitrosourea-based chemotherapy such as carmustine, lomustine, fotemustine either alone or in combination with other drugs were the standard chemotherapeutic regimens for recurrent malignant glioma, with Overall response rates ranged from 10 to $30 \%$ depending on the pathology type and type of assessment the response rate. There is a good example of relatively cost-effective regimen. There was no clear survival benefit when comparing nitrosourea-based chemotherapy PCV with temozolomide $(6.7 \text { v } 7.2 \text { months, respectively })^{5}$.

In this study, the response rate was $5 \%$ partial response with is relatively low than the previous reported response rate and I think the reason for that is related to using a maximum of CT scan for assessment in the previous studies, not MRI as we did. And this low response rate was consistent for recent data on Lomustine as a single agent with only $4 \%$ partial response ${ }^{16}$. While third generation nitrosourea fotemustine had $14 \%$ partial response ${ }^{17}$.

This study confirms the value of giving palliative nitrosourea-based chemotherapy to patients with progressive high grade glioma. In spite of it is a retrospective work, we had adequate data to reach our aim of this study. Even with relatively old drugs, we had a significant improvement of overall survival. And we have to say that the value was significant for both glioblastoma multiform and anaplastic astrocytoma.

\section{CONCLUSIONS}

In conclusions, this study proved that patients with progressive high grade glioma benefit from nitrosourea-based chemotherapy. The benefit was in the form of significant improvement in overall survival. Nitrosourea-based chemotherapy should be considered a reasonable option in recurrent high grade glioma.

\section{Disclosure:}

The authors report no conflicts of interest in this work.

\section{REFERENCES}

1. Stupp R, Mason WP, van den Bent MJ, et al. Radiotherapy plus concomitant and adjuvant temozolomide for glioblastoma. N Engl J Med. 2005; 352: 987-996.

2. Scoccianti S, Magrini SM, Ricardi U, et al: Radiotherapy and temozolomide in anaplastic astrocytoma: a retrospective multicenter study by the Central Nervous System Study Group of AIRO (Italian Association of Radiation Oncology). Neuro-Oncology. 2012; 14(6): 798-807.

3. Hau P, Baumgart U, Pfeifer K, et al. Salvage therapy in patients with glioblastoma: is there any benefit?. Cancer. 2003; 98: 2678 . 
4. Dinnes J, Cave C, Huang S, et al. A rapid and systematic review of the effectiveness of temozolomide for the treatment of recurrent malignant glioma. $\mathrm{Br} \mathrm{J}$ Cancer. 2002; 86: 501-505.

5. Brada M, Stenning S, Gabe R, et al. Temozolomide Versus Procarbazine, Lomustine, and Vincristine in Recurrent High-Grade Glioma. J Clin Oncol. 2010; 28: 4601-4608.

6. Friedman HS, Prados MD, Wen PY, et al. Bevacizumab Alone and in Combination With Irinotecan in Recurrent Glioblastoma. J Clin Oncol. 2009; 27: 4733-4740.

7. Wen PY, Macdonald DR, David A. Reardon DA, et al. Updated Response Assessment Criteria for High-Grade Gliomas: Response Assessment in Neuro-Oncology Working Group. J Clin Oncol. 2010; 28:1963-1972.

8. Keles GE, Lamborn KR, Chang SM, et al. Volume of residual disease as a predictor of outcome in adult patients with recurrent supratentorial glioblastoma multiforme who are undergoing chemotherapy. J. Neurosurg. 2004; 100: 41-46.

9. Hou LC, Veeravagu A, Hsu AR, et al. Recurrent glioblastoma multiforme: a review of natural history and management options. Neurosurg Focus. 2006; 20:E5.

10. Combs SE, Widmer V, Thilmann C. Stereotactic radiosurgery (SRS). Treatment options for recurrent glioblastoma multiforme (GBM). Cancer. 2005; 104: 2168-2173.

11. Souhami L, Seiferheld W, Brachman D, et al. Randomized comparison of stereotactic radiosurgery followed by conventional radiotherapy with carmustine to conventional radiotherapy with carmustine for patients with glioblastoma multiforme: report of Radiation Therapy Oncology Group 93-05 protocol. Int J Radiat Oncol Biol Phys. 2004; 60(3): 853-60.

12. Yung WK, Prados MD, Yaya-Tur R, et al. Multicenter phase II trial of temozolomide in patients with anaplastic astrocytoma or anaplastic oligoastrocytoma at first relapse. Temodal Brain Tumor Group. J Clin Oncol. 1999; 17: 2762-2771.

13. Perry JR, Belanger K, Mason WP, et al. Phase II Trial of Continuous Dose-Intense Temozolomide in Recurrent Malignant Glioma: RESCUE Study. J Clin Oncol. 2010; 28: 2051-2057.

14. Perry JR, Rizek P, Cashman R, et al. Temozolomide rechallenge in recurrent malignant glioma by using a continuous temozolomide schedule: The rescue approach. Cancer. 2008; 113: 2152-2157.

15. Wick A, Pascher C, Wick W, et al. Rechallenge with temozolomide in patients with recurrent gliomas. J Neurol. 2009; 256(5): 734-741.

16. Wick W, Puduvalli VK, Chamberlain MC, et al. Phase III Study of Enzastaurin Compared With Lomustine in the Treatment of Recurrent Intracranial Glioblastoma. J Clin Oncol. 2010; 28: 1168-1174.

17. Paccapelo A, Lolli I, Fabrini3 MG, et al. A retrospective pooled analysis of response patterns and risk factors in recurrent malignant glioma patients receiving a nitrosourea-based chemotherapy. Journal of Translational Medicine. 2012; 10: 90. 